IMTC 2006 - Instrumentation and Measurement

Technology Conference

Sorrento, Italy 24-27 April 2006

\title{
Performance Assessment of Wireless Communication Networks through Cross-Layer Measurements
}

\author{
Leopoldo Angrisani ${ }^{1}$, Michele Vadursi ${ }^{2}$ \\ ${ }^{1}$ Dipartimento di Informatica e Sistemistica ${ }^{2}$ Dipartimento di Ingegneria Elettrica \\ Università degli Studi di Napoli Federico II, \\ Via Claudio, 21 - 80125 Napoli, Italy \\ Phone : +390817683170 - Fax : +390812396897-Email : \{angrisan; mvadursi\}@unina.it
}

\begin{abstract}
Assessing the overall performance of wireless communication networks is of key importance for optimal management and planning. With special regard to wireless networks operating in an unlicensed band, evaluating overall performance mainly implies facing coexistence issues, associated with the contemporaneous presence of true and interfering signals at physical layer. This task is difficult, if not prohibitive, to be fulfilled only on the basis of single-layer measurements; a partial perspective of network behavior would, in fact, be gained. At this concern, a cross-layer approach is presented hereinafter. It provides for several measurements, to be concurrently carried out at different layers through an innovative automatic station. It aims at correlating the values major physical layer quantities (e.g. channel power, signal-to-noise ratio) exhibit to those characterizing key network/transport layer parameters (e.g. packet loss ratio, one-way delay). A first step towards a full characterization of how the effects of a problem, experienced either at physical layer or network/transport layer, propagates along the whole protocol stack can thus be taken. Details on the procedure underlying the proposed approach, as well as the realized measurement station, are given with reference to an application example.
\end{abstract}

Keywords - Cross-layer measurements, Interference measurements, Wireless communication networks, Wi-Fi, Packet loss ratio measurements, One way delay measurements, Coexistence issues.

\section{INTRODUCTION}

Overall performance of any communication network is strictly connected to the functionalities of each layer in its ISO/OSI (International Organization for Standardization/ Open System Interconnect) stack. In particular, it depends on the characteristics of data link and physical layers that manage data flowing through all physical channels involved. While on wired channels proper signal integrity is assured by mechanical, electrical and protocol characteristics of the aforementioned layers, on wireless channels this is not true. Unpredictable and uncontrollable signal degradation can severely affect data transmission, and ultimately compromise the performance perceived by the final user, generally quantified through objective network/transport layer parameters, such as packet loss ratio, timing jitter, and one way delay.

High reliability of typical network/transport layer protocols makes the overall performance of wireless or hybrid communication networks significantly depend on signal integrity on the wireless channel. However, an exhaustive characterization of physical layer does not prove appropriate to determine how possible signal degradation affects key net- work/transport layer parameters, and ultimately the perceived performance. Dually, a full characterization at network/transport layer, although very close to an overall performance evaluation, does not provide any useful instrument to fix possible problems at the vulnerable physical layer.

So, research activity aimed at going beyond a single layer measurement approach in order to establish the influence of signal degradation on the performance of typical wireless or hybrid networks seems to be relevant and should be encouraged. This is particularly true for communication networks operating in the unlicensed ISM (Industrial Scientific Medical) band, like Wi-Fi, Bluetooth, ZigBee, and WiMAX networks. Coexistence issues, mainly related to the superposition of desired and interfering signals in the same band, have to be of major concern in such cases [1]-[4].

The work aims at correlating the values major physical quantities in the wireless channel exhibit to those characterizing key network/transport layer parameters. In a word, the work can be said to take advantage of cross-layer measurements, intended to assess the performance of a network layer as a function of that of another or several other ones. This represents one of the first attempts to experimentally put into relation physical layer measures with higher layer parameters. At the current state of art, cross-layer performance evaluation of wireless systems is carried out in a simulated fashion. In some cases, measurements are performed, but only for a single layer [5]-[8]. On the contrary, the work provides for several measurements to be concurrently carried out at different layers, through an innovative automatic station.

\section{PROPOSED APPROACH}

A cross-layer approach is proposed to correlate major physical layer measures to the values of key network/transport layer parameters. With regard to the physical layer, channel power and signal-to-interference power ratio are enlisted. Concerning network/transport layer, the attention is focused on packet loss ratio (PLR), timing jitter, and oneway delay (OWD), which have direct influence on the quality perceived by users in a variety of heterogeneous applications. The description is given with reference to an application example, which involves a real test-bed compliant with IEEE $802.11 \mathrm{~b}$ standard (Wi-Fi). As already stated, a significantly challenging problem is represented by physical layer vulnerability. To properly address it, an experimental test-bed is 
used, which allows degrading signal integrity by introducing interference of known characteristics in the wireless channel. The test-bed is, in fact, located inside a semi-anechoic chamber.

\section{A. Measurement station}

A suitable measurement station has been set up, which is sketched in Fig.1. Several experimental tests have been carried out in the semi-anechoic chamber of the Department of Electrical Engineering at the University of Naples Federico II. In such environment, a total control of interference is, in fact, possible. The only interfering signal that is present on the communication channel is emitted from a signal generator suitably commanded, whereas possible uncontrolled interference from outside the chamber does not affect the communication. Specifically, the measurement station consists of (i) three hosts; (ii) an 802.11 Access Point (AP) D-link DI-624+; (iii) a signal generator Rhode\&Schwarz SML03 (9 kHz - 3.3 $\mathrm{GHz}$ frequency range, with pulse modulation capability); which provides for sinusoidal interference; (iv) another signal generator Agilent Technologies E4403B $(9 \mathrm{kHz}-3.0 \mathrm{GHz}$ frequency range), which provides for modulated interference; (v) an arbitrary waveform generator (AWG) Agilent Technologies $33120 \mathrm{~A}$ (15 MHz maximum frequency); (vi) a microwave horn antenna Amplifier Research AT4002A (0.8 $\mathrm{GHz}-5 \mathrm{GHz}$ frequency range); (vii) an omnidirectional antenna EM-6865 (frequency range $2 \mathrm{GHz}-18 \mathrm{GHz}, 10.16 \mathrm{~cm}$ diameter); (viii) a spectrum analyzer Anritsu MS2687B (9 $\mathrm{kHz}-30 \mathrm{GHz}$ input frequency range, up to $20 \mathrm{MHz}$ resolution bandwidth); (ix) a digital storage oscilloscope (DSO) Agilent Technologies 54833D (1 GHz bandwidth, $4 \mathrm{GS} / \mathrm{s}$ sampling frequency, 8.2 megasample memory depth); (x) an EIA232to-TTL converter, which makes a pulse generated on the serial port of the sender host available as trigger signal for both the spectrum analyzer and DSO. The dashed box in Fig.1 encloses instruments that are inside the semi-anechoic chamber. The three generators, spectrum analyzer, DSO, and one of the hosts, which is the processing and control unit of the measurement station, are all interconnected via an IEEE 488 standard interface bus.
The signal inside the chamber is captured by the omnidirectional antenna, which is connected to the spectrum analyzer through a coaxial cable. Besides providing the power spectrum of the signal captured by the antenna, the spectrum analyzer can be utilized in zero span mode to attain the evolution versus time of signal envelope power. Moreover, it can act as a downconverter to an intermediate frequency equal to $66 \mathrm{MHz}$; thanks to this feature, the DSO can acquire the evolution versus time of the downconverted signal, whose significant spectral content is totally included inside the DSO bandwidth.

\section{B. Measurement procedure}

Communication occurs between a pair of hosts, in the following referred to as Host 1 and Host 2. Host 1 is connected via a wired $100 \mathrm{Mbps}$ link to the AP, which represents its default gateway. Wireless communication, compliant with IEEE 802.11b standard, actually takes place between AP and Host 2 , which are inside the chamber. An active measurement approach is in particular adopted. Synthetic UDP (User Datagram Protocol) traffic is generated through D-ITG (Distributed Internet Traffic Generator) [9], whose architecture allows measuring several Quality of Service (QoS) parameters at both sender and receiver sides, and reporting a complete digest of measured parameters over the entire measurement time.

Controlled interference is emitted by the two signal generators, which are outside the semi-anechoic chamber and feed the microwave horn antenna located inside. Several types of both continuous and bursty interfering signals are taken into consideration. To emit bursty interference, in particular, pulse modulation capability of the signal generators is exploited, while AWG, used as trigger source, is commanded to generate an either periodic or frequency modulated square wave signal.

As stated above, channel power and SIR measurements are carried out at physical layer. Channel power is measured through the spectrum analyzer, in the absence of interference. It is worth noting that spectral analysis on Wi-Fi signals through a spectrum analyzer places some constraints, due to

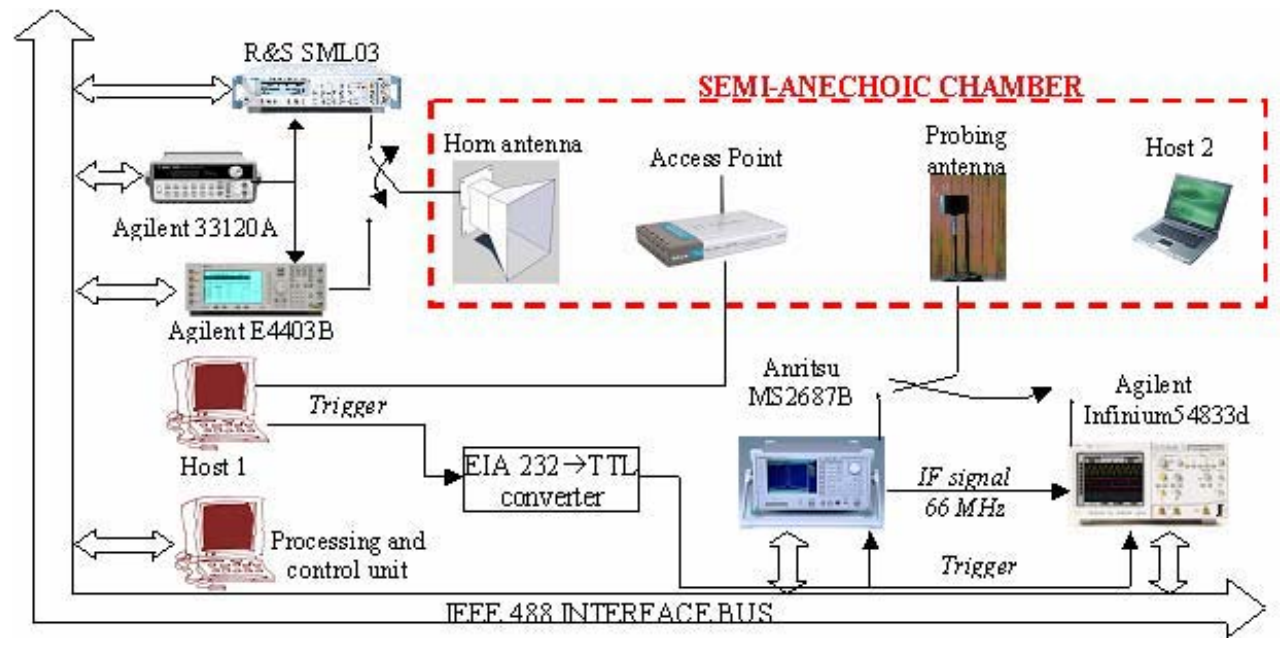

Fig.1. Measurement station 
the limited duration of transmitted packets. Transmission of long packets, in fact, requires no more than few milliseconds, and the minimum allowed sweep time covers several packets. A synchronization with transmitted packets would prevent from sweeping when no signal is present in the channel; signal power spectrum could therefore be reconstructed by joining power spectrum segments of successive packets. This solution is implemented by setting the spectrum analyzer in gate mode, and performing the sweep over the selected frequency span at intervals synchronized with the transmission of packets. With respect to the version presented in [9], in fact, D-ITG has been provided with a triggering feature, already exploited in [10], which is very useful to the purpose at hand. In particular, when the sender starts the transmission of each packet, a voltage pulse is generated on a certain pin of its serial port. The EIA232-to-TTL converter has been designed by the authors to use the pulse emitted on the serial port of the sender host as trigger signal for the spectrum analyzer (and/or the DSO). An external trigger source is, in fact, needed, because beacon frames, periodically generated by the $\mathrm{AP}$, could induce wrong triggers. Although this technique has the disadvantage that measurement time grows inversely with packet transmission rate, it allows analyzing signal power spectrum with the sensibility typical of a spectrum analyzer, rather than performing an FFT (Fast Fourier Transform)based analysis. Interference power is similarly measured through the spectrum analyzer, in order to evaluate signal-tointerference power ratio $(S I R)$.

\section{EXPERIMENTAL RESULTS}

Experiments have been conducted in the presence of different types of interfering signals, such as sinusoidal, FSK (Frequency Shift Keying) and Bluetooth signals, and taking into consideration both continuous and bursty interference.

\section{A. Sinusoidal interference}

For the sake of brevity, only results related to PLR, in the presence of bursty sinusoidal interference characterized by random occurring times, are given.

For an easier comprehension of the results, the following parameters have to be clarified:

$-T_{O N}$ is the duration of a single burst of interference.

$-T$ is the average time interval between two successive bursts. $-d$ (duty cycle $)=T_{O N} / T$.

$-S_{M}$ is the power of the continuous interfering sinusoidal sig-

Table I. PLR as a function of interference level, for different packet rates $(d=0.1)$.

\begin{tabular}{|c|c|c|c|c|c|c|c|c|c|c|c|c|c|c|c|c|}
\hline & \multicolumn{16}{|c|}{ Interference power [dBm] } \\
\hline & $\mathbf{S}_{\mathbf{M}}$ & -49.3 & -46.2 & -45.6 & -44.1 & -43.1 & -42.1 & -41.1 & -40.2 & -39.2 & -38.2 & -37.2 & -34.1 & -33.6 & -33.1 & -32.0 \\
\hline & $S_{\text {BURST }}$ & -59.3 & -56.2 & -55.6 & -54.1 & -53.1 & -52.1 & -51.1 & -50.2 & -49.2 & -48.2 & -47.2 & -44.1 & -43.6 & -43.1 & -42.0 \\
\hline \multirow{2}{*}{$\begin{array}{c}250 \\
\mathrm{pkt} / \mathrm{s}\end{array}$} & SIR & 14.4 & 11.3 & 10.7 & 9.2 & 8.3 & 7.2 & 6.2 & 5.3 & 4.3 & 3.3 & 2.3 & -0.8 & -1.3 & -1.8 & -2.9 \\
\hline & PLR & $2.7 \%$ & $4.1 \%$ & $6.3 \%$ & $24 \%$ & $30 \%$ & $31 \%$ & $35 \%$ & $38 \%$ & $35 \%$ & $35 \%$ & $36 \%$ & $38 \%$ & $37 \%$ & $34 \%$ & $32 \%$ \\
\hline \multirow{2}{*}{$\begin{array}{c}750 \\
\text { pkt } / \mathrm{s}\end{array}$} & SIR & 14.2 & 11.1 & 10.5 & 9.0 & 8.0 & 7.0 & 6.0 & 5.1 & 4.1 & 3.1 & 2.1 & -1.0 & -1.5 & -2.0 & -3.1 \\
\hline & PLR & $58 \%$ & $63 \%$ & $65 \%$ & $67 \%$ & $70 \%$ & $74 \%$ & $76 \%$ & $77 \%$ & $78 \%$ & $78 \%$ & $78 \%$ & $78 \%$ & $78 \%$ & $78 \%$ & $79 \%$ \\
\hline \multirow{2}{*}{$\begin{array}{l}1000 \\
\text { pkt/s }\end{array}$} & SIR & 14.7 & 11.6 & 11.0 & 9.5 & 8.5 & 7.5 & 6.5 & 5.6 & 4.6 & 3.6 & 2.6 & -0.5 & -1.0 & -1.5 & -2.6 \\
\hline & PLR & $70 \%$ & $73 \%$ & $75 \%$ & $72 \%$ & $79 \%$ & $81 \%$ & $83 \%$ & $83 \%$ & $84 \%$ & $84 \%$ & $84 \%$ & $84 \%$ & $84 \%$ & $84 \%$ & $83 \%$ \\
\hline
\end{tabular}

Table II. PLR as a function of interference level, for different packet rates $(d=0.15)$.

\begin{tabular}{|c|c|c|c|c|c|c|c|c|c|c|c|c|c|c|c|c|}
\hline & \multicolumn{16}{|c|}{ Interference power $[\mathrm{dBm}]$} \\
\hline & $\mathbf{S}_{\mathbf{M}}$ & -46.8 & -45.9 & -45.3 & -43.8 & -42.8 & -41.9 & -40.9 & -39.9 & -38.9 & -37.9 & -36.9 & -33.9 & -33.3 & -32.8 & -31.8 \\
\hline & $\mathbf{S}_{\text {BURST }}$ & -55.1 & -54.4 & -53.6 & -52.1 & -51.1 & -50.1 & -49.1 & -48.2 & -47.2 & -46.2 & -45.2 & -42.1 & -41.6 & -41.1 & -40.1 \\
\hline \multirow{2}{*}{$\begin{array}{c}250 \\
\mathrm{pkt} / \mathrm{s}\end{array}$} & SIR & 10.1 & 9.2 & 8.7 & 7.2 & 6.2 & 5.2 & 4.2 & 3.2 & 2.2 & 1.2 & 0.2 & -2.8 & -3.3 & -3.9 & -4.9 \\
\hline & PLR & $7.8 \%$ & $34 \%$ & $38 \%$ & $48 \%$ & $50 \%$ & $55 \%$ & $56 \%$ & $53 \%$ & $57 \%$ & $58 \%$ & $56 \%$ & $55 \%$ & $54 \%$ & $57 \%$ & $57 \%$ \\
\hline \multirow{2}{*}{$\begin{array}{c}750 \\
\mathrm{pkt} / \mathrm{s}\end{array}$} & SIR & 9.9 & 9.0 & 8.5 & 7.0 & 6.0 & 5.0 & 4.0 & 3.1 & 2.1 & 1.1 & 0.1 & -3.0 & -3.5 & -4.0 & -5.1 \\
\hline & PLR & $65 \%$ & $70 \%$ & $72 \%$ & $76 \%$ & $79 \%$ & $82 \%$ & $83 \%$ & $84 \%$ & $86 \%$ & $85 \%$ & $85 \%$ & $85 \%$ & $85 \%$ & $85 \%$ & $85 \%$ \\
\hline \multirow{2}{*}{$\begin{array}{l}1000 \\
\text { pkt/s }\end{array}$} & SIR & 10.5 & 9.6 & 9.0 & 7.5 & 6.5 & 5.5 & 4.5 & 3.6 & 2.6 & 1.6 & 0.6 & -2.5 & -3.0 & -3.5 & -4.5 \\
\hline & PLR & $73 \%$ & $77 \%$ & $78 \%$ & $81 \%$ & $84 \%$ & $86 \%$ & $88 \%$ & $89 \%$ & $88 \%$ & $89 \%$ & $90 \%$ & $89 \%$ & $89 \%$ & $89 \%$ & $89 \%$ \\
\hline
\end{tabular}

Table III. PLR as a function of interference level, for different packet rates $(d=0.2)$.

\begin{tabular}{|c|c|c|c|c|c|c|c|c|c|c|c|c|c|c|c|c|}
\hline & \multicolumn{16}{|c|}{ Interference power [dBm] } \\
\hline & $\mathbf{S}_{\mathrm{M}}$ & -49.3 & -46.3 & -45.7 & -44.2 & -43.2 & -42.2 & -41.4 & -40.7 & -40.1 & -39.5 & -39.1 & -34.2 & -33.7 & -33.2 & -32.1 \\
\hline & $S_{\text {BURST }}$ & -56.3 & -53.3 & -52.7 & -51.2 & -50.2 & -49.2 & -48.4 & -47.7 & -47.1 & -46.5 & -46.1 & -41.2 & -40.7 & -40.2 & -39.2 \\
\hline \multirow{2}{*}{$\begin{array}{c}250 \\
\text { pkt/s }\end{array}$} & SIR & 11.4 & 8.4 & 7.8 & 6.3 & 5.3 & 4.3 & 3.5 & 2.8 & 2.2 & 1.6 & 1.2 & -3.7 & -4.3 & -4.7 & -5.9 \\
\hline & PLR & 12 & 19 & 27 & 33 & 39 & 44 & 54 & 61 & 64 & 63 & 64 & 65 & 66 & 64 & 65 \\
\hline \multirow{2}{*}{$\begin{array}{c}750 \\
\text { pkt } / \mathrm{s}\end{array}$} & SIR & 11.2 & 8.2 & 7.6 & 6.1 & 5.1 & 4.1 & 3.3 & 2.6 & 2.0 & 1.4 & 1.0 & -3.9 & -4.4 & -4.9 & -6.0 \\
\hline & PLR & 71 & 75 & 78 & 80 & 83 & 85 & 88 & 88 & 88 & 87 & 89 & 88 & 88 & 88 & 88 \\
\hline \multirow{2}{*}{$\begin{array}{l}1000 \\
\mathrm{pkt} / \mathrm{s}\end{array}$} & SIR & 11.7 & 8.7 & 8.1 & 6.6 & 5.6 & 4.6 & 3.8 & 3.1 & 2.5 & 1.9 & 1.5 & -3.4 & -3.9 & -4.4 & -5.5 \\
\hline & PLR & 79 & 82 & 83 & 85 & 88 & 89 & 90 & 91 & 91 & 91 & 91 & 92 & 92 & 92 & 91 \\
\hline
\end{tabular}


nal, hereinafter named peak power, which is measured through the spectrum analyzer.

$-S_{B U R S T}=d \cdot S_{M}$ is the average power of interference.

$-C_{P}$ is the channel power of the Wi-Fi signal, which is measured through the spectrum analyzer in the absence of interference.

$-S I R=C_{P} / S_{B U R S T}$ is the signal-to-interference ratio.

Interfering signal carrier frequency has been fixed at 2.427 $\mathrm{GHz}$, and PLR has been evaluated over a 60 second transmission, in order to be able to confidently assume $S_{B U R S T}$ as the average power of interference. Moreover, values of $S_{M}$ and $C_{P}$, measured through the spectrum analyzer, have been averaged over one hundred successive measurements to mitigate repeatability problems power measurements on (wideband) RF signals suffer from [11]. All power values have been expressed in $\mathrm{dBm}, S I R$ in $\mathrm{dB}$, PLR in percentage relative terms, and packet rate and length have been given in packet/s and byte, respectively. Table I, II, and III report PLR for different interfering signal power levels, and different transmission packet rates. They are related to a duty cycle $d$ equal respectively to $0.1,0.15$, and 0.2 .

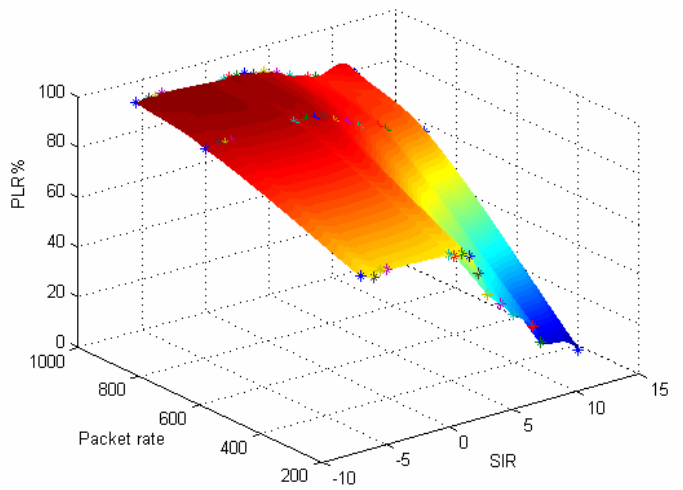

Fig.2. PLR as a function of packet rate and $\operatorname{SIR}(d=0.2)$

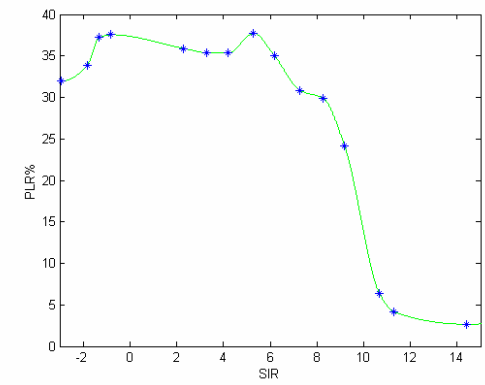

Fig.4.a. PLR vs. SIR (rate $=250 \mathrm{pkt} / \mathrm{s} ; d=0.1$ ).

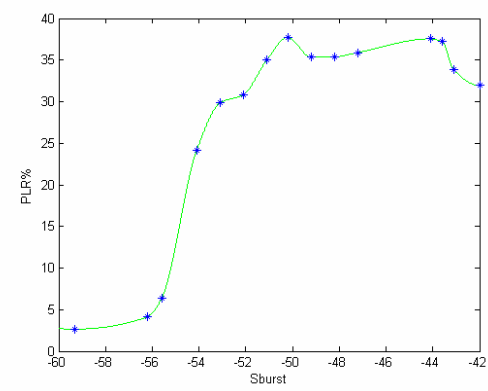

Fig.4.d. PLR vs. $\mathrm{S}_{\mathrm{BURST}}(\mathrm{rate}=250 \mathrm{pkt} / \mathrm{s} ; d=0.1)$.

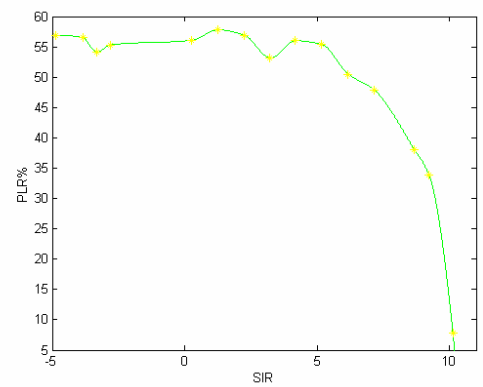

Fig.4.b. PLR vs. SIR (rate $=250 \mathrm{pkt} / \mathrm{s} ; d=0.15$ ).

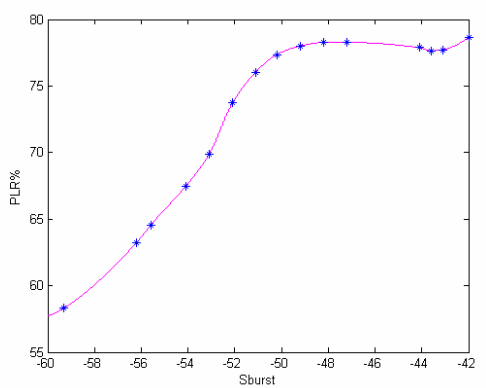

Fig.4.e. PLR vs. $\mathrm{S}_{\mathrm{BURST}}(\mathrm{rate}=750 \mathrm{pkt} / \mathrm{s} ; d=0.1)$
Fig. 2 and Fig. 3 provide a three-dimensional picture of PLR evolution versus transmission and interference parameters. Specifically, Fig.2 shows PLR as a function of packet rate and $S I R$, for a given duty cycle $(d=0.2)$, whereas Fig. 3 gives PLR versus duty cycle and $S_{B U R S T}$ for a packet rate equal to $750 \mathrm{pkt} / \mathrm{s}$. A more detailed representation of measurement results can be gained from Fig.4, which provides the evolution of PLR as a function of, respectively, $S_{B U R S T}$ (Fig.4.a,b,c) and SIR (Fig.4.d,e,f), for different combinations of duty cycle and transmission packet rate.

From the analysis of the results, the following considerations can be drawn.

- Since channel power of Wi-Fi signal does not vary significantly with transmission rate, SIR and $S_{B U R S T}$ are equivalent figures of merit in the analyzed cases.

- All plots are characterized by a similar evolution. A threshold $S I R$ value can be singled out, which separates a variation region, in which the lower the SIR (or the higher the $S_{B U R S T}$ ) the worse the PLR, from a saturation region, in which the worst PLR is reached.

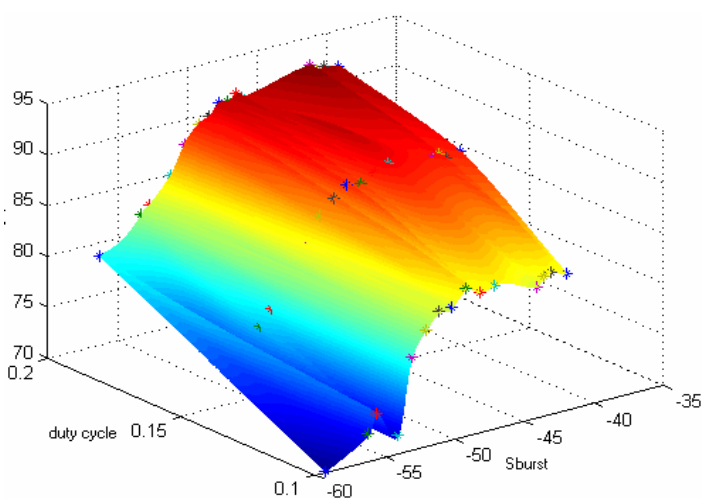

Fig.3. PLR as a function of $d$ and $S_{B U R S T}$ (packet rate=750 pkt/s).

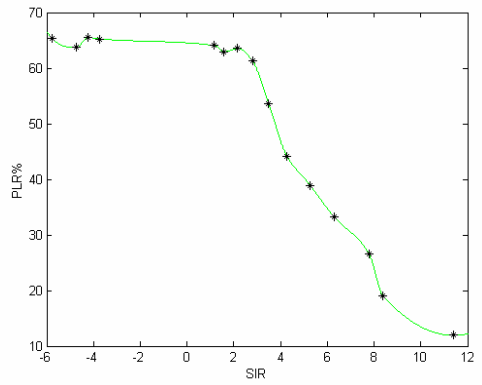

Fig.4.c. PLR vs. SIR (rate $=250 \mathrm{pkt} / \mathrm{s} ; d=0.2$ ).

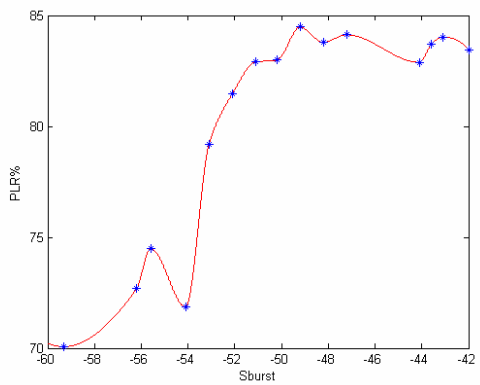

Fig.4.f. PLR vs. $\mathrm{S}_{\mathrm{BURST}}(\mathrm{rate}=1000 \mathrm{pkt} / \mathrm{s} ; d=0.1)$. 

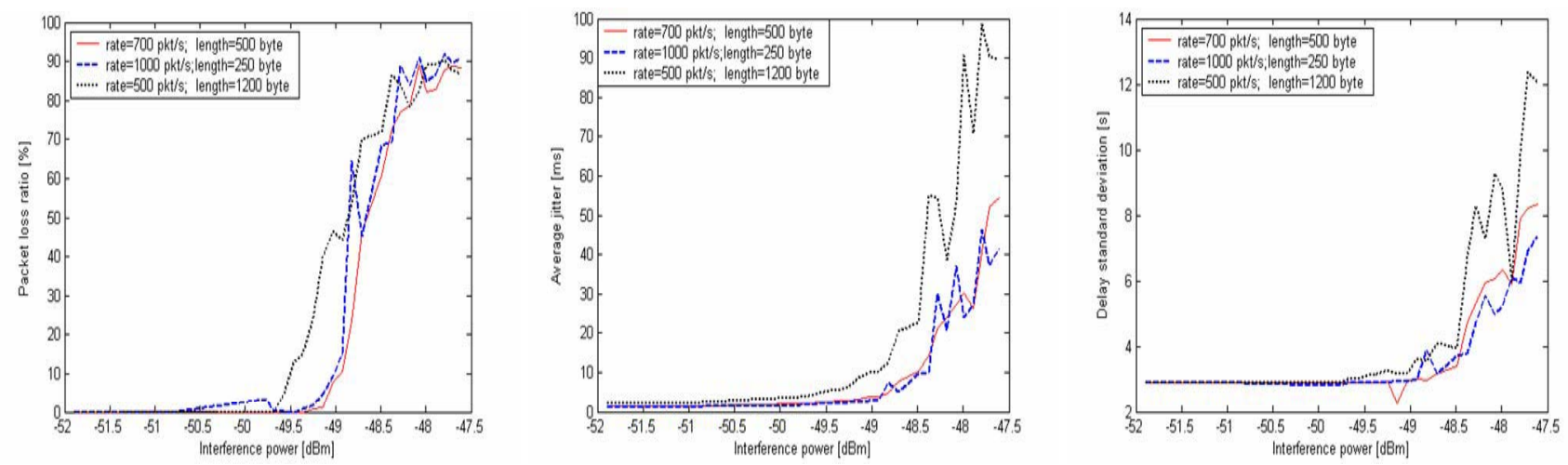

Fig.5. Evolution of (a) PLR, (b) average jitter, and (c) delay experimental standard deviation, versus 2-FSK interference level, for different values of packet length and rate.

- Maximum PLR is not necessarily equal to $100 \%$, but it depends on packet transmission rate and $T$. Once SIR has gone below the aforementioned threshold, given a certain value of $T$ (or packet transmission rate), PLR is not affected by further SIR degradation, but mainly depends on packet transmission rate (or $T$ ).

\section{B. FSK and Bluetooth interference}

A set of experiments has been carried out in the presence of 2-FSK interfering signals, characterized by frequency deviation equal to $170 \mathrm{kHz}$ and bit rate equal to $1 \mathrm{Mbps}$. Different values of carrier frequency and average power of the 2FSK interfering signal have been considered. In all cases, carrier frequency has fallen within the Wi-Fi signal bandwidth (the AP has been configured to utilize Wi-Fi channel number 4, i.e. carrier frequency equal to $2.427 \mathrm{GHz}$ ). Experiments have also been repeated for different values of packet rate and packet length. Host 1 and Host 2 have been synchronized through Network Time Protocol (NTP) [12].

Fig.5 shows the results achieved with continuous 2-FSK interference, when interfering and Wi-Fi signal have the same carrier frequency, for different values of interfering signal level, and transmitted packet length and rate. Results are expressed in terms of PLR (Fig.5.a), average jitter $J$ (Fig.5.b), which is defined as

$$
J=\sum_{i=1}^{n-1} \frac{\left|D_{i}\right|}{n-1}
$$

where $n$ is the number of transmitted packets and

$$
D_{i}=\left(R_{i+1}-R_{i}\right)-\left(S_{i+1}-S_{i}\right)
$$

is the difference between interarrival $\left(R_{i+1}-R_{i}\right)$ and interdeparture times $\left(\mathrm{S}_{i+1}-S_{i}\right)$, and delay experimental standard deviation.

Please note that interference power levels have been chosen in order to prevent from compromising Wi-Fi communication. Values of interference power higher than those shown in Fig. 5 would, in fact, make establishing $802.11 \mathrm{~b}$ communication impossible, as the transmitter would sense the channel and find it continuously busy.

The results have shown that degradation increases (as expected) upon the interference level's increasing. No significant dependence of degradation on packet size or length can be highlighted, although Wi-Fi transmission seems to be slightly more sensitive to interference when longer packets are transmitted. What is notable, it is possible to single out a threshold for interference power value, over which the three parameters taken into consideration jump up very high values. With regard to the transmission of 500 byte packets at a rate equal to $700 \mathrm{packet} / \mathrm{s}$, for example, less than $1 \mathrm{~dB}$ increase in interference power can make PLR move from $0 \%$ to more than $70 \%$ ! Similarly, average jitter is 20 -fold increased, from less than $3 \%$ to more than $40 \%$, upon a $1.5 \mathrm{~dB}$ increase of interference power. A further increase of interference power, lower than $1 \mathrm{~dB}$, would make Wi-Fi communication impossible, as already stated.

Fig. 6 shows the results achieved with continuous Bluetooth interference, with random payload, when interfering and Wi-Fi signal have the same carrier frequency, for different values of interfering signal power, and transmitted packet length and rate. Evolution versus interference power of each parameter is practically the same as that experienced with 2FSK interference. Wi-Fi communication is not affected by interference as long as interference power level is below a certain power threshold; when interference power is increased over such threshold, performance degrades very rapidly, until communication is totally precluded. Similar outcomes have been experienced when the carrier frequency of the interfering signal is 5 or $10 \mathrm{MHz}$ greater than that of the Wi-Fi signal, but the power values in correspondence of which interference degrades the network performance are significantly lower.

\section{Comments}

By analyzing the experimental results, and considering the standard Wireless Medium Access Control (MAC) and Physical Layer (PHY) specifications, the reason for such behavior can be inferred. Stations regularly perform Clear Channel Assessment (CCA) to check the status (busy/idle) of the channel. Interference has practically no effect as long as its power does not pass a threshold $\Theta$ over which the AP starts to consider the physical channel busy. When interference power goes over $\Theta$, transmission is blocked, and a queue overflow is experienced at the AP. Such two-state behavior is, however, only ideal. A transition region can be sin- 

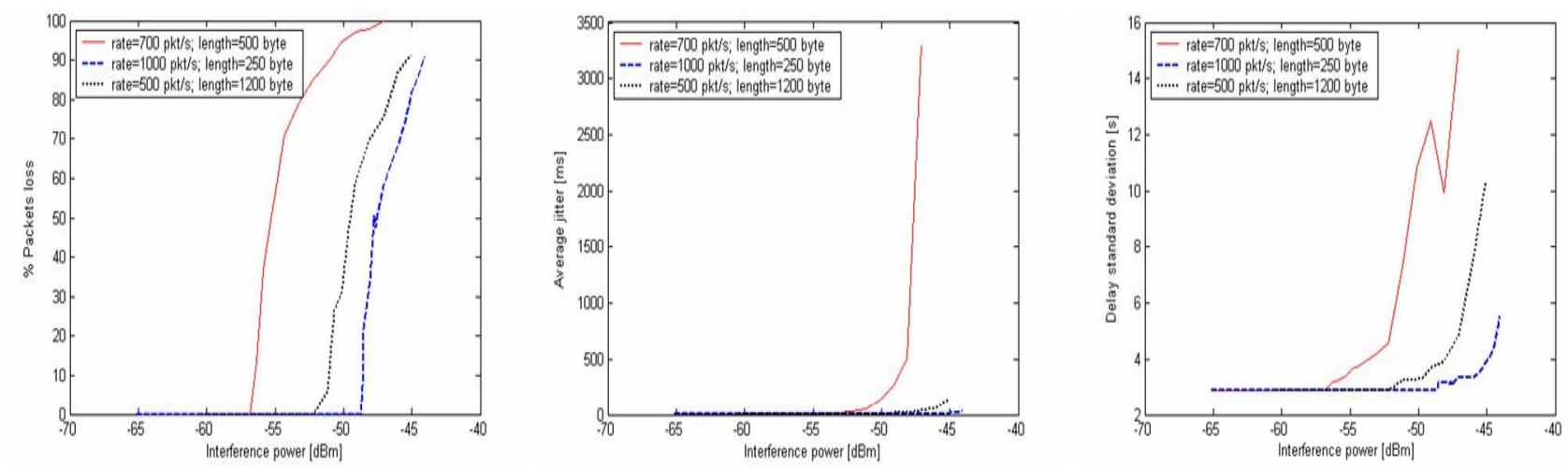

Fig.6.Evolution of (a) PLR, (b) average jitter, and (c) delay experimental standard deviation, versus Bluetooth interference level, for different values of packet length and rate.

gled out, whose width plausibly depends on receiver sensitivity, for power values very close to $\Theta$, where the channel is sensed sometimes busy, sometimes idle, and transmission goes by fits and starts. This explains why performance passes from a satisfying to a degraded status very quickly. An alternative explanation could be that interference damages packets, which are therefore retransmitted, even more than once, according to the MAC protocol, thus causing queue overflow at AP side. Besides being not realistic, as Wi-Fi networks are robust with respect to narrowband interference (whereas the behavior under discussion has been observed with sinusoidal interference, too), such hypothesis has been discarded after analyzing the results of further experiments. In particular, single packets have been transmitted and interference has been triggered immediately after the packet has occupied the channel; the evolution versus time of the signal in the wireless channel, made possible by the DSO, has shown that MAC-layer acknowledgement is one SIFS (Short InterFrame Space) distant from the end of the transmitted packet, which means that no retransmission has occurred, proving that interference has not damaged the packet. In conclusion, although Wi-Fi modulation is robust with regard to narrowband interference, its MAC protocol strongly suffers from the presence of interference, because of the sensitivity of carrier sensing mechanism, which leads to queue overflow at sender side and consequent possible packet loss.

\section{CONCLUSION}

A cross-layer approach has been presented to assess the performance of wireless networks operating in the ISM band. An application example related to a Wi-Fi network in the presence of in-channel interference ha been presented. Thanks to the original proposed approach, and to the suitable measurement station set up for the scope, it has been possible to correlate the values major physical layer quantities exhibit to those characterizing key network/transport layer parameters. The cross-layer approach has also been decisive to infer the cause of the observed performance degradation, discarding hypotheses that have not been supported by the experimental evidence. Results show that when interference level is below a certain threshold, the channel is sensed as idle and interference does not affect Wi-Fi communication. On the contrary, when interference level passes such threshold, the transmission is blocked as the channel is sensed busy; packets are consequently lost, due to buffer overflow, and not because damaged by interference. Future research activities will investigate the relation between AP receiver sensitivity and transition region width, and extend the application of experimental test-bed to more complex networks, as well as networks compliant with different standards.

\section{REFERENCES}

[1] N. Golmie, N. Chevrollier, O. Rebala, "Bluetooth and WLAN coexistence: challenges and solutions", IEEE Wireless Communications, Vol.10, no.6, Dec. 2003, pp. 22- 29.

[2] Y. Matsumoto, M. Takeuchi, K. Fujii, A. Sugiura, Y. Yamanaka, "Performance analysis of interference problems involving DS-SS WLAN systems and microwave ovens", IEEE Trans. on Electromagnetic Compatibility, Vol.47, no.1, Feb. 2005, pp. 45-53.

[3] K. Takaya, Y. Maeda, N. Kuwabara, "Experimental and theoretical evaluation of interference characteristics between 2.4-GHz ISM-band wireless LANs," IEEE Int. Symp. on Electromagnetic Compatibility, 1998, Vol.1, 24-28 Aug 1998, pp:80-85.

[4] A. Sikora, V. F. Groza, "Coexistence of IEEE802.15.4 with other Systems in the 2.4 GHz-ISM-Band," Proc. of IEEE Instr. and Meas. Tech. Conf. IMTC 2005, Ottawa (Canada), May 2005, pp.1786-1791.

[5] G. Xylomenos, G. C. Polyzos, "TCP and UDP performance over a wireless LAN", Proc. of INFOCOM 99, vol.2, pp. 439-446.

[6] J. C. Amaro, R. P. Lopes, "Performance analysis of a wireless MAN," Network Computing and Applications, 2001.

[7] D. Moltchanov, Y. Koucheryavy, J. Harju, "Cross-layer analytical modeling of wireless channels for accurate performance evaluation," Proc. of QoFiS'2004, Barcelona, Spain, Oct. 2004, pp. 194-203.

[8] S. Pollin, B. Bougard. G. Lenoir, B. Van Poucke, L. Van der Perre, F. Catthoor, I. Moerman "Cross-layer exploration of link adaptation in wireless LANs with TCP traffic," IEEE Symp. on Communications and Vehicular Technology (SCVT'03), Eindhoven (NL), Nov. 2003.

[9] http://www.grid.unina.it/software/ITG

[10] L. Angrisani, S. D’Antonio, M. Esposito, M. Vadursi, “Techniques for Available Bandwidth Measurement in IP Networks: a Performance Comparison," Computer Networks (Elsevier), Vol.50, Issue 3, 22 February 2006, pp.332-349.

[11] L.Angrisani, M.D'Apuzzo, M.Vadursi, "Power measurements in digital wireless communication systems through parametric spectral estimation," Proc. of IEEE Instrum. and Measur. Technol. Conf., IMTC 2005, 17-19 May 2005, pp.2323-2328.

[12] http://www.ntp.org/ 